\title{
One-step generation of a targeted knock-in calf using the CRISPR-Cas9 system in bovine zygotes
}

\author{
Joseph R. Owen', Sadie L. Hennig ${ }^{1}$, Bret R. McNabb², Tamer A. Mansour ${ }^{2,3}$, Justin M. Smith ${ }^{1}$, Jason C. Lin',
} Amy E. Young ${ }^{1}$, Josephine F. Trott ${ }^{1}$, James D. Murray ${ }^{1,2}$, Mary E. Delany ${ }^{1}$, Pablo J. Ross ${ }^{1}$ and Alison L. Van Eenennaam ${ }^{1 *}$ (1)

\begin{abstract}
Background: The homologous recombination (HR) pathway is largely inactive in early embryos prior to the first cell division, making it difficult to achieve targeted gene knock-ins. The homology-mediated end joining (HMEJ)-based strategy has been shown to increase knock-in efficiency relative to HR, non-homologous end joining (NHEJ), and microhomology-mediated end joining (MMEJ) strategies in non-dividing cells.

Results: By introducing gRNA/Cas9 ribonucleoprotein complex and a HMEJ-based donor template with $1 \mathrm{~kb}$ homology arms flanked by the $\mathrm{H} 11$ safe harbor locus gRNA target site, knock-in rates of $40 \%$ of a $5.1 \mathrm{~kb}$ bovine sexdetermining region Y (SRY)-green fluorescent protein (GFP) template were achieved in Bos taurus zygotes. Embryos that developed to the blastocyst stage were screened for GFP, and nine were transferred to recipient cows resulting in a live phenotypically normal bull calf. Genomic analyses revealed no wildtype sequence at the H11 target site, but rather a $26 \mathrm{bp}$ insertion allele, and a complex $38 \mathrm{~kb}$ knock-in allele with seven copies of the SRY-GFP template and a single copy of the donor plasmid backbone. An additional minor $18 \mathrm{~kb}$ allele was detected that looks to be a derivative of the $38 \mathrm{~kb}$ allele resulting from the deletion of an inverted repeat of four copies of the SRY-GFP template.

Conclusion: The allelic heterogeneity in this biallelic knock-in calf appears to have resulted from a combination of homology directed repair, homology independent targeted insertion by blunt-end ligation, NHEJ, and rearrangement following editing of the gRNA target site in the donor template.

This study illustrates the potential to produce targeted gene knock-in animals by direct cytoplasmic injection of bovine embryos with gRNA/Cas9, although further optimization is required to ensure a precise single-copy gene integration event.
\end{abstract}

Keywords: CRISPR, Knock-in, Gene editing, Bovine, Embryos, Bos taurus

\footnotetext{
* Correspondence: alvaneenennaam@ucdavis.edu

'Department of Animal Science, University of California - Davis, Davis, CA,

USA

Full list of author information is available at the end of the article
}

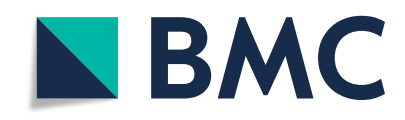

(-) The Author(s). 2021 Open Access This article is licensed under a Creative Commons Attribution 4.0 International License, which permits use, sharing, adaptation, distribution and reproduction in any medium or format, as long as you give appropriate credit to the original author(s) and the source, provide a link to the Creative Commons licence, and indicate if changes were made. The images or other third party material in this article are included in the article's Creative Commons licence, unless indicated otherwise in a credit line to the material. If material is not included in the article's Creative Commons licence and your intended use is not permitted by statutory regulation or exceeds the permitted use, you will need to obtain permission directly from the copyright holder. To view a copy of this licence, visit http://creativecommons.org/licenses/by/4.0/. The Creative Commons Public Domain Dedication waiver (http://creativecommons.org/publicdomain/zero/1.0/) applies to the data made available in this article, unless otherwise stated in a credit line to the data. 


\section{Background}

The targeted integration of large DNA segments into livestock genomes has remained challenging since the production of the first random integrant transgenic livestock were reported 35 years ago [1]. Typically, targeted insertions have been performed in cell lines, followed by somatic cell nuclear transfer cloning (SCNT) [2]. However, SCNT is associated with high rates of both pregnancy and perinatal loss. There are few reports of embryo-mediated targeted insertions in livestock, and they frequently result in mosaic embryos with more than two alleles resulting from independent editing events following the first cleavage division [3]. Mosaic animals are problematic in uniparous large animals with long generation interval (2 years for cattle), as it requires several years to produce a non-mosaic animal through conventional breeding.

Attempts have been made to increase the efficiency of performing targeted gene insertions utilizing the homologous recombination (HR) pathway [4], which is primarily restricted to actively dividing cells (S/G2-phase) and only becomes highly active towards the end of the first round of DNA replication [5]. However, these have been largely unsuccessful in bovine embryos [6], and often result in mosaic animals. A homology mediated end-joining (HMEJ)-based strategy was found to be an efficient gene knock-in strategy in mouse and monkey embryos [7], as well as chicken primordial germ cells [8]. Multiple repair pathways are thought to be involved in mediating a gene knock-in using this method. Previously, we found that the use of a HMEJ repair template to target an insertion to the $\mathrm{X}$ chromosome increased the knock-in frequency in bovine embryos as compared to a traditional HR template [9], and that more than a third of knock-in blastocysts analyzed were non-mosaic with precise integrations [10]. Blunt end ligation of cleaved donor template by homology independent insertion was also observed, more frequently in male than female embryos, but no integration of the donor plasmid backbone was ever detected [10].

The objective of this study was to insert a $1.8 \mathrm{~kb}$ DNA segment, the sex-determining region of the $Y$ chromosome $(S R Y)$ gene, into a targeted location in the bovine genome. This gene, typically located on the mammalian $Y$ chromosome, is expressed in early embryonic development and results in a cascade of factors necessary for initiating male gonadal development and shutting down development of the female gonad [11]. We wished to investigate whether the inheritance of the bovine SRY gene would be sufficient to trigger the male developmental pathway in XX bovine embryos. Male calves are desirable as sale animals in beef cattle production systems because they have greater feed efficiency than females and reach market readiness at a heavier weight.
Given the time and expense to perform bovine embryo transfers, and the subsequent nine-month gestation required to produce a calf, it was necessary to confirm the presence of the $S R Y$ insertion prior to embryo transfer to a recipient cow. The diagnostic value of invasive preimplantation biopsies of cells derived from the trophectoderm of blastocysts as a means of screening for knock-ins is decreased in genome edited embryos [12] due to the potential for mosaicism [13]. In the current study a safe harbor locus, H11 on Chromosome 17, was targeted as the insertion site and a fluorescent reporter protein was employed to allow for the non-invasive screening of embryos to identify those carrying the gene insertion prior to embryo transfer.

\section{Results}

Production of a gene knock-in bull calf

To generate the targeted knock-in bull, a HMEJ donor template containing the $1.8 \mathrm{~kb}$ bovine sex-determining region $\mathrm{Y}(S R Y)$ promoter and coding sequencing [14], the $1.3 \mathrm{~kb} G F P$ reporter transgene coding sequencing with Simian virus 40 (SV40) promoter was designed. It included $1 \mathrm{~kb}$ homology arms flanked on the outside by the gRNA target site [15] of the H11 safe harbor locus [16] on bovine chromosome 17 (5.1 kb "complete template”, Fig. 1a). Genomic safe harbors can incorporate exogenous pieces of DNA and permit their predictable function, but these edits do not pose adverse health risks to the host organism [17].

Approximately 200 in vitro fertilized bovine zygotes were microinjected with gRNA/Cas9 ribonucleoprotein complex and HMEJ-template at $6 \mathrm{~h}$ post insemination (hpi), which is prior to the initiation of zygote DNA replication at 11-15 hpi. Twenty-two embryos reached the blastocyst stage, and nine (40\%) showed green fluorescence indicating successful transgene integration (Figs. 1b-d). These nine embryos were non-surgically transferred to synchronized recipients. The remaining 13 blastocysts were genotyped and sequenced and 11 were found to carry mutations at the $\mathrm{H} 11$ locus. One recipient (Tag 3113) was confirmed pregnant by transrectal ultrasonography at day 35 of gestation, and the phenotypic sex was likewise determined at day 68 by the location of the genital tubercle, indicating a male phenotype (Fig. 2a). A healthy $50 \mathrm{~kg}$ bull calf was born in April 2020 (Fig. 2b).

DNA was extracted from placenta, calf blood, and the fibroblast cell line derived from the calf, and analyzed for SRY-GFP knock-in, as well as genotypic sex. PCR and Sanger sequencing revealed a biallelic edit that included both the complete SRY-GFP template and a 26 base pair (bp) insertion into the H11 locus (Fig. 2c), in addition to an XY genotype (Fig. 2d). 


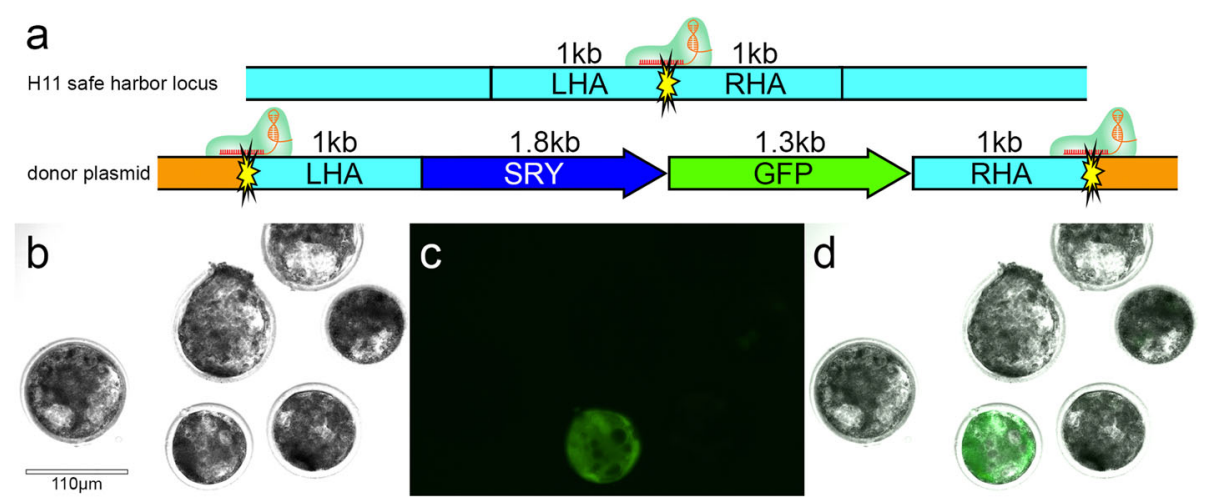

Fig. 1 The CRISPR-mediated knock-in of bovine embryos by homology mediated end joining (HMEJ). We utilized the HMEJ donor template design with the green fluorescent protein reporter gene to develop a non-invasive screening method of bovine blastocysts to visualize knock-in embryos. a schematic representation of the complete template in the pUC19 plasmid (orange). Yellow starburst = gRNA target site at the $\mathrm{H} 11$ locus on chromosome 17 with gRNA/Cas9 ribonucleoprotein complex bound; LHA = left homology arm; SRY = sex-determining region Y; GFP = green fluorescent protein; RHA = right homology arm; kb = kilobase $\mathbf{b}$ day seven microinjected bovine blastocysts under bright field $\mathbf{c}$ a filter specific for eGFP fluorescence showing a fluorescent blastocyst, and $\mathbf{d}$ merge of bright field and fluorescent image

\section{Sequence analysis of the knock-in allele}

Given that the PCR results from the samples taken from tissue types of trophectodermal and mesodermal origin were identical, DNA extracted from blood was used for Illumina whole-genome sequencing (paired-end, $150 \mathrm{bp}$ ) on a NovaSeq 6000 sequencer (Novogene, USA) to 268X coverage. Raw reads were mapped to the complete template on chromosome 17 (Fig. 3a), the $26 \mathrm{bp}$ insertion allele (Fig. 3b), and the HMEJ donor pUC19 plasmid backbone (Fig. 3c). There was a $4 \mathrm{X}$ increase in reads that aligned to the complete template compared to the $26 \mathrm{bp}$ insertion. In addition, some reads aligned to the pUC19 plasmid backbone (Fig. 3c). This suggested integration of the donor plasmid backbone, in addition to the intended knock-in template, as was observed previously $[18,19]$.

To investigate the insertions more fully, PacBio longread sequencing was generated from the same blood sample. From all PacBio reads, we identified 314 sequences with some similarity to the complete template, the $26 \mathrm{bp}$ insert, the donor plasmid backbone, and/or the H11 locus on chromosome 17. Then, a reference sequence was generated which included the complete ARS-UCD1.2 bovine genome sequence [20], the plasmid backbone and the complete template sequences. Mapping the 314 candidate reads, we detected no wild-type $\mathrm{H} 11$ allele and 3 insertion alleles. The $26 \mathrm{bp}$ insertion into the wild-type H11 allele that was detected by Sanger sequencing (Fig. 3b) was supported by 49 long reads. The other 2 alleles each included 1 copy of the plasmid backbone sequence (Fig. 3c) with multiple copies of the complete template (Fig. 3a, d). The larger $\sim 38 \mathrm{~kb}$ allele had around 50X coverage and consisted of 7 copies of the complete template along with 1 copy of the plasmid backbone. The smaller $18 \mathrm{~kb}$ allele had 3 copies of the complete template in addition to 1 copy of the plasmid backbone and was unambiguously supported by only 5 long reads. This allele is identical to the larger complex allele but missing the middle 4 copies of the complete template sequence (Fig. 3d).

\section{Fluorescence in situ hybridization (FISH) of SRY}

The $S R Y$ insert was consistently detected near the q arm terminus of one chromosome providing additional evidence for the insertion into a single location (Fig. 4). The chromosome size and type, i.e., smaller-sized acrocentric, align with that expected for Bos taurus (BTA) chromosome 17 and this insert map location was cytogenetically confirmed by dual color FISH experiments employing a BTA 17 specific BAC. The SRY signal detected at the knock-in location was likely amplified given the presence of multiple copies of the gene inserted at the H11 target site as shown by sequencing. Conversely, a faint $S R Y$ signal was only occasionally detected on the Y chromosome, and only following significant signal amplification by image analysis. This result is likely due to the non-repetitive and small size of the single copy SRY gene in its native state, coupled with the resolution-scale of FISH.

\section{Discussion}

The birth of this calf represents the first successful targeted integration of a large DNA segment produced by embryo-mediated genome editing in cattle. Although we achieved a $40 \%$ knock-in rate as determined by GFP expression in blastocysts, only $22(11 \%)$ of the $\sim 200$ microinjected embryos developed to the blastocyst stage. We previously observed a significant reduction in blastocyst development following microinjection of editing regents into MII oocytes (10.2\%) and presumptive zygotes 6 hpi (17.6\%), as compared to non-injected controls $(29.3 \%)$ [10, 15]. Additionally, only one of the nine embryos transferred resulted in a live birth. This is a low 


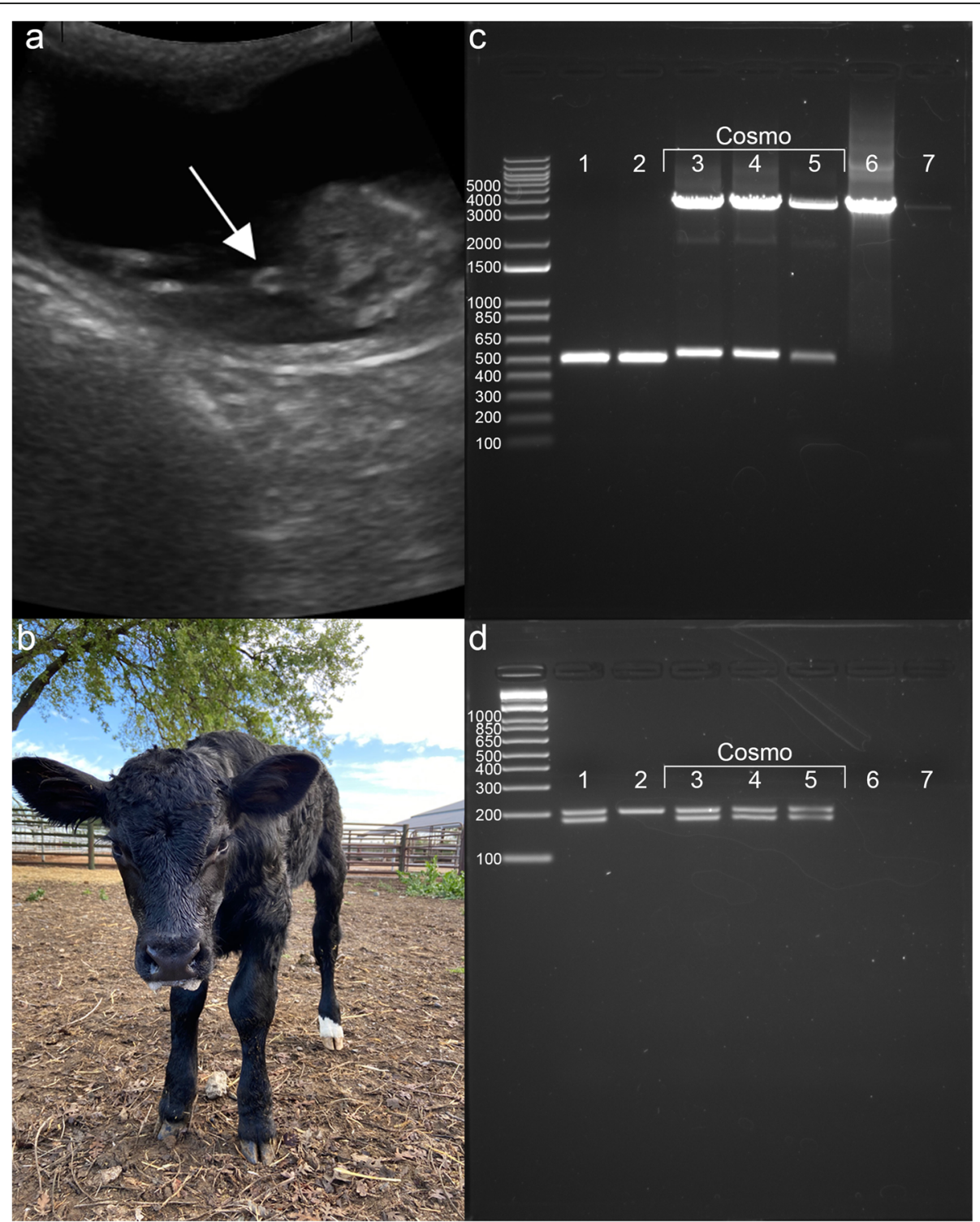

Fig. 2 Development of a targeted knock-in bull calf. We monitored and analyzed the development of the SRY-GFP knock-in bull calf produced by cytoplasmic injection of a homology mediated end joining donor template and the CRISPR-Cas9 system in bovine zygotes. a ultrasound of the day 68 fetus revealing the male genital tubercle (arrow) caudal to the umbilicus indicating a male phenotype, $\mathbf{b}$ the SRY-GFP knock-in bull calf (Cosmo) at 2 days of age, $\mathbf{c}$ Analysis of SRY-GFP knock-in by the polymerase chain reaction (PCR). DNA was extracted from three tissue types: placental cotyledons (trophectodermal origin), blood and fibroblast cells (mesodermal origin). The donor plasmid was used as the positive control and water was used as the negative control. Expected band sizes: wild type 520 bp, SRY-GFP knock-in 3721 bp. The lower band from the calf runs higher than wild type due to the 26 bp insertion, and d Genotypic sex. Expected band sizes: female 208 bp; male 189 bp \& 208 bp. lane $1=$ wild type male; lane 2 = recipient female (3113); lane 3 = Cosmo placenta; lane $4=$ Cosmo blood; lane $5=$ Cosmo fibroblast; lane $6=$ plasmid; lane 7 = water

success rate (11.1\%), although it is a small sample size, and on average only $27 \%$ of recipients receiving conventional in vitro produced (IVP) embryos result in a live calf [21]. Further experiments with a larger number of embryos will be also be required to determine if briefly screening blastocysts for GFP affected viability. It is known that UV light can be harmful to living embryos, although others have reported viable pregnancies following short exposure of bovine blastocysts to blue light to screen for GFP expression [22]. More generally, the current low efficiencies of precise targeted integration of large DNA segments, embryo development and live births of non-mosaic animals limits the utility of embryo-mediated gene knockins in cattle breeding programs $[6,23]$.

The only other group to report a bovine embryomediated targeted gene knock-in used TALENs and a 

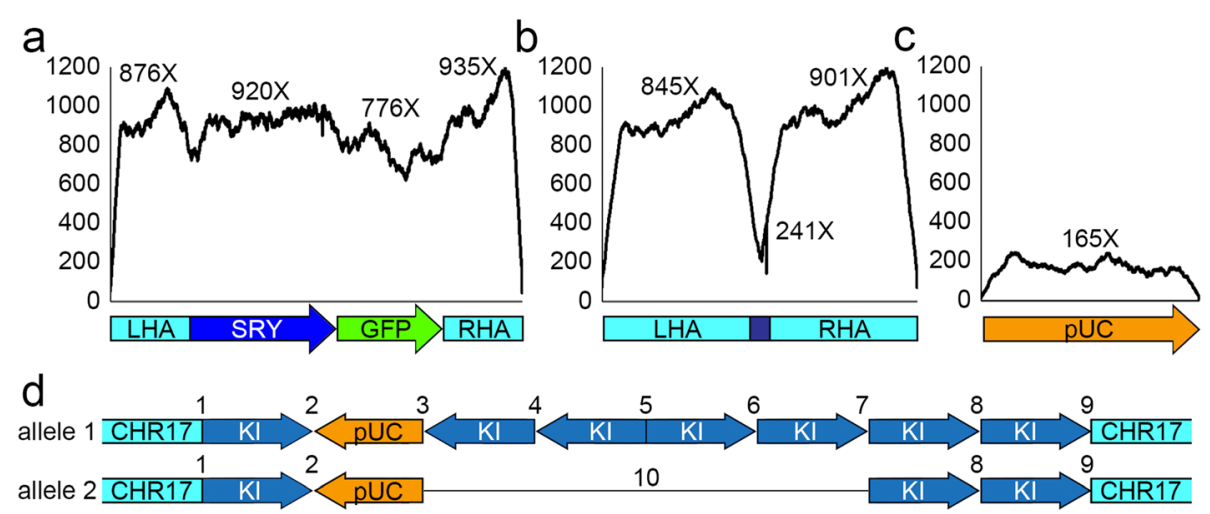

e
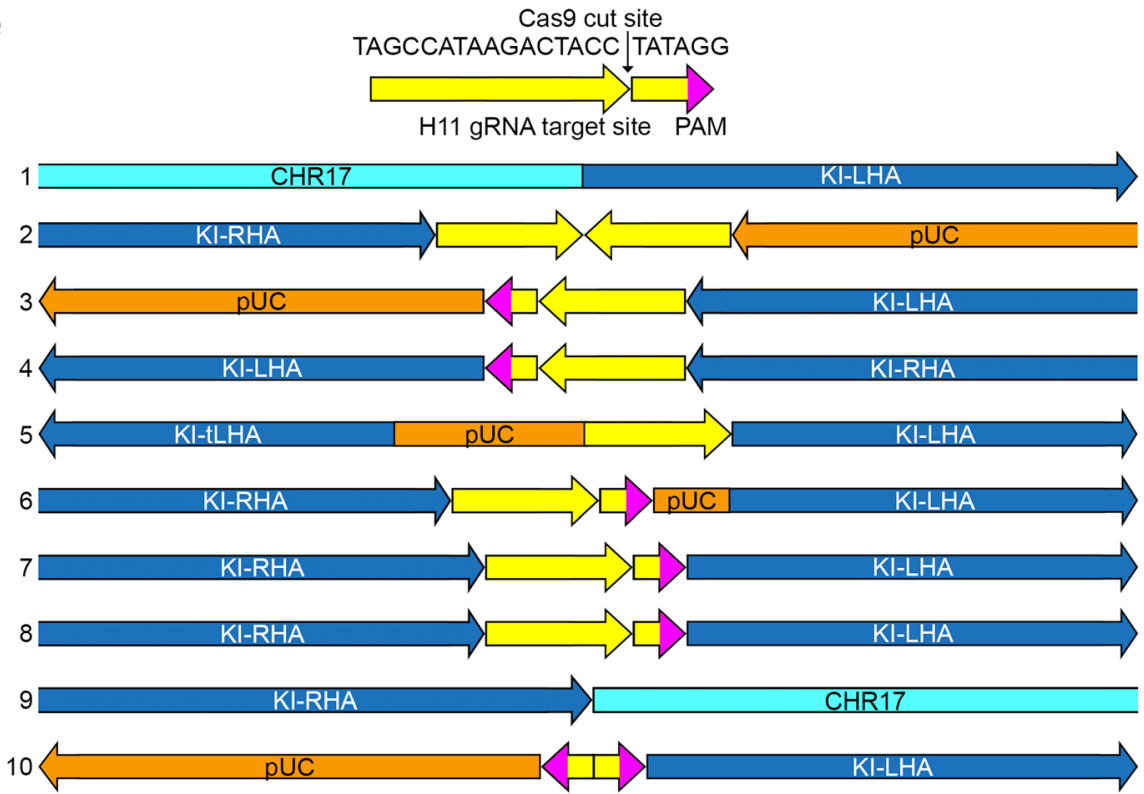

Fig. 3 Identification of allelic sequence at the $\mathrm{H} 11$ target site. The coverage depth was calculated for the mapped alignment of Illumina NovaSeq whole genome sequencing reads to the expected knock-in, the Sanger sequenced 26 bp knock-in allele, and the pUC19 donor plasmid backbone. Reads were then used to identify the junction sites between the insertions. a coverage depth of reads aligned to the complete $5.1 \mathrm{~kb}$ SRY-GFP template $\mathbf{b}$ coverage depth of reads aligned to the $26 \mathrm{bp}$ insertion, $\mathbf{c}$ coverage depth of reads aligned to the $2.7 \mathrm{~kb}$ pUC19 donor plasmid backbone (orange), and $\mathbf{d}$ the $38 \mathrm{~kb}$ and $18 \mathrm{~kb}$ complex insertions, and e gRNA target site and Cas9 cut site (yellow) at the H11 locus on chromosome 17, and schematic representation of the $38 \mathrm{~kb}$ and $18 \mathrm{~kb}$ complex allele insertion junctions (1-10). LHA = left homology arm; $\mathrm{SRY}=$ sex-determining region $\mathrm{Y} ; \mathrm{GFP}=$ green fluorescent protein; $\mathrm{RHA}=$ right homology arm; $\mathrm{pUC}=\mathrm{pUC19}$ donor plasmid backbone; $\mathrm{CHR} 17=$ genomic region outside homology arms on chromosome 17; KI = complete $5.1 \mathrm{~kb}$ SRY-GFP template; tLHA = truncated left homology arm; PAM = protospacer adjacent motif

single-strand oligonucleotide (ssODN) HR donor template to introduce a targeted $9 \mathrm{bp}$ deletion in the bovine lactoglobulin $(L G B)$ gene [24]. In that experiment, the editing reagents were introduced into 1511 bovine zygotes at $18 \mathrm{hpi}$. Of these, 234 (15\%) developed to grade 7 or 8 embryos, of which $50(21 \%)$ were confirmed to carry the 9 bp $L G B$ deletion by biopsies of 10-15 cells derived from the trophectoderm of blastocysts. Of these, 13 were transferred to generate three $(23 \%)$ live births, of which one calf died shortly after birth.

In the current experiment, no H11 wild-type allele was amplified by PCR (Fig. 2c). There were 11 short read sequences (6 after deduplication) that supported H11 wild-type sequence in the more than $250 \mathrm{X}$ sequence coverage, but no single long read contained wild-type H11 sequence. The $26 \mathrm{bp}$ and $38 \mathrm{~kb}$ insertion alleles were both represented at around 50X coverage in the long-read sequencing data. Collectively, these data suggest that a biallelic edit at the zygote stage, one of which was repaired by NHEJ resulting in a $26 \mathrm{bp}$ insertion, and the other $38 \mathrm{~kb}$ complex allele knock-in which appears to have resulted from a combination of homology directed repair, homology independent targeted insertion by blunt-end ligation, and rearrangement following editing of the gRNA target site in the donor template. 


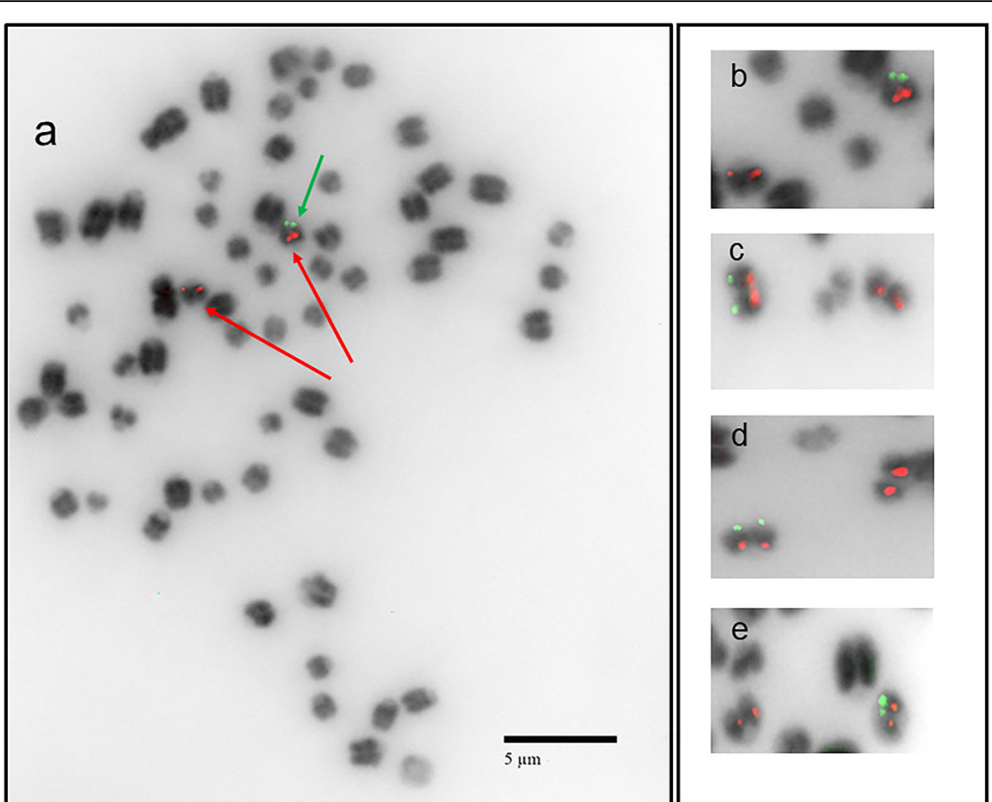

Fig. 4 One BTA 17 homolog identified as the map location for the SRY insert in the CRISPR-targeted knock-in bull calf by dual color fluorescence in situ hybridization (FISH). FISH with the donor plasmid (SRY-GFP Anti-Digoxigenin-Fluorescein) as the probe identified one acrocentric chromosome with a positive signal at the q-arm terminal region confirming a single insertion site into the knock-in calf genome. The acrocentric was identified as BTA 17 utilizing a chromosome-specific centromere-proximal probe labelled with Red dUTPs (CHORI BAC 371i17, see Methods). The q-arm terminal location of the SRY green signal found opposite to the centromere proximal BAC red signal compliments the expected insertion location at the safe-harbor as per the sequencing results. Male and female controls (no insertion) were also examined using the same SRY probe with no signal(s) observed (data not shown). a Diploid mitotic metaphase chromosome spread from a fibroblast culture derived from the SRY-GFP knock-in bull shows a normal karyotype, $2 n=60$ with a single SRY-GFP positive signal (green arrow) on one of the two BTA17 chromosomes (red arrows) b enlarged SRY-GFP knock-in BTA 17 chromosome (red and green signals) along with the other BTA 17 chromosome (red signal only) from cell depicted in (a) and $\mathbf{c}$, d, e enlarged BTA 17 chromosomes from other cells illustrate the reproducibility of the FISH results. Chromosomes shown in b-e were all enlarged to the same degree

Multiple copies of the complete template in both forward and reverse orientations in the $38 \mathrm{~kb}$ allele was not expected with the HMEJ-mediated strategy, and was not observed in our previous study using this approach [10]. Such concatenation is more typical of homology independent targeted insertion (HITI) [25]. In the case of this bull calf, it appears the far left and right homology arms were repaired by HR as there is no H11 gRNA target site footprint at the boundary where the left homology arm meets the $5^{\prime}$ wild-type genomic sequence of bovine chromosome 17 , or where the right homology arms meets the 3' wild-type sequence. Many of the other junctions between inserts contain partial H11 gRNA target sequences (Fig. 3e). This suggests that the RNP complex cut the donor plasmid at the H11 gRNA target sites and the resulting double-stranded fragments integrated by blunt end ligation. The repair mechanism for junctions 5 and 6 in the $38 \mathrm{~kb}$ complex allele is less apparent as both sequences include a short plasmid backbone sequence, $56 \mathrm{bp}$ and $9 \mathrm{bp}$, respectively. The overall complexity of this insertion allele suggests a potential concern associated with knock-in strategies which involve flanking the homology arms with sgRNA target sites. In this study it appears Cas9 cleavage of these target sites contributed to the integration of the multiple copies of the donor template in various orientations, and one copy of the plasmid backbone, rather than the precise integration that was predicted.

A minor $18 \mathrm{~kb}$ complex allele was also detected at approximately one tenth the read coverage of the 38 $\mathrm{kb}$ allele. The only difference in the DNA sequence between the $38 \mathrm{~kb}$ and $18 \mathrm{~kb}$ complex alleles was the loss of four complete templates (two in the reverse direction and 2 in the forward direction) (Fig. 3d). This indicates that the $18 \mathrm{~kb}$ allele, which was present in all 3 tissue types analyzed (placenta, fibroblasts, and blood; data not shown), may represent a deletion derivative of the larger $38 \mathrm{~kb}$ allele, rather than a separate editing event. The inverted repeat nature of the sequence that was deleted, i.e., two complete $S R Y$ GFP templates in the reverse direction followed immediately by two complete SRY-GFP templates in the forward direction, may indicate instability in the $38 \mathrm{~kb}$ insertion allele. 
While multiple copies of the donor template and a single copy of the donor backbone were inserted into the target location, there was no off-target insertion of the donor template or donor backbone detected. This was demonstrated by the lack of short or long reads containing donor template or donor backbone sequence in any region outside the $\mathrm{H} 11$ locus. In addition, the only FISH signals detected for the presence of the insert were that of a single homolog at the q-arm end of BTA 17, which aligns with the H11 locus which is located $\sim 3 \mathrm{~kb}$ from the terminus of BTA 17.

Strategies aimed at avoiding unwanted plasmid backbone integration in genome editing include using single stranded DNA (ssDNA) repair templates, which have a significantly reduced frequency of unintended genomic integration. However, the primary success with targeting a knock-in of embryos using ssDNA has been through attempting allelic conversions, such as small insertions, deletions or single nucleotide polymorphisms. Each of these cases was performed using ssODNs of varying length ranging from 35 to $120 \mathrm{bp}$ [26-28]. The largest integration performed using ssDNA was a 1368 bp insert using a $\sim 1.5 \mathrm{~kb}$ ssODN in mouse embryos in a method called Easi-CRISPR [29]. Attempts to insert larger segments of DNA using ssODN through microinjection or electroporation have been unsuccessful in embryos [30].

In this experiment we chose to use a fluorescent marker to identify blastocysts with the $S R Y$ knock-in, and to target an autosomal safe harbor locus following our previous unsuccessful attempts to obtain live calves when targeting the $S R Y$ to a $\mathrm{X}$ chromosome locus and screening for knock-ins using embryo biopsy [10]. It is possible that the inclusion of the SV40 promoter to drive the expression of the GFP gene could result in the silencing of the adjacent SRY gene, as has been commonly observed with the hypermethylation of the cytomegalovirus (CMV) promoter. The SV40 promoter has been found to maintain more steady levels of expression when stably integrated in mammalian cells as compared to the CMV promoter [31]. A recent paper reported transgenic cattle expressing GFP driven by the human elongation factor $1 \alpha$ promoter showed stable GFP expression over 6 years and F2 germline transmission without gene silencing [32]. It is also possible that the presence of multiple copies of the transgene in the complex alleles in the current study may also lead to repeat-induced gene silencing.

Although the addition of the GFP gene technically made the knock-in bull calf transgenic, the United States Food and Drug Administration regulates all genomic alterations in animals as new animal drugs [33], irrespective of whether a transgene is present [34]. As we had no intention for this genetically altered research line to enter the food chain, the inclusion of the GFP transgene in the donor template design to provide a rapid, non- invasive screening method to ensure that only knock-in embryos were transferred to recipient cows, outweighed the fact that it was a transgene.

\section{Conclusions}

The low efficiency of direct HR repair in zygotes, especially for the introduction of large DNA sequences, remains an obstacle for the incorporation of useful genetic variants into livestock genetic improvement programs. The HMEJ-based strategy used in this study did increase the efficiency of HR editing in zygotes, but it also resulted in multiple homology independent blunt-end insertions, including one copy of the donor plasmid backbone. Unintended homology independent insertions may not be problematic for some research applications; however this potential is untenable for embryo-mediated therapeutic applications where precise integration is requisite, and would also pose potential challenges for the regulatory approval of food animal applications.

\section{Methods}

\section{Experimental design}

The objective of this study was to produce a targeted gene knock-in Bos taurus bull by direct cytoplasmic microinjection of single-cell bovine embryos using a donor template containing the bovine $S R Y$ promoter and coding sequence, the $g f p$ coding sequence with SV40 promoter utilizing the HMEJ-approach. Once a pregnancy was established, the phenotypic sex was determined by transrectal ultrasound and following birth, genotypic sex was determined, and the on-target and off-target integration of the donor template was evaluated using short and long read whole genome sequencing technology.

\section{Embryo production}

Ovaries were obtained from cull Bos taurus cows of unknown breed at a local processing plant and transported in warm sterile saline at temperature of $35-37^{\circ} \mathrm{C}$. Oocyte-cumulus-complexes (COCs) were aspirated from follicles using a vacuum aspiration system and cultured in groups of $50 \mathrm{COCs}$ in $500 \mu \mathrm{L}$ of BO-IVM culture media (IVF Biosciences, Falmouth, UK) for $18 \mathrm{~h}$ at $38.5^{\circ} \mathrm{C}$ in a humidified $5 \% \mathrm{CO}_{2}$ incubator. COCs were then washed and transferred in groups of 25 to $60 \mu \mathrm{L}$ drops of SOF-IVF media [35] with $2 \times 10^{6}$ sperm per $\mathrm{mL}$ and covered in mineral oil. Sperm and COCs were incubated for $6 \mathrm{~h}$ at $38.5{ }^{\circ} \mathrm{C}$ in a humidified $5 \% \mathrm{CO}_{2}$ incubator. Presumptive zygotes were then denuded by light vortex and transferred to $25 \mu \mathrm{L}$ of BO-IVC culture media (IVF Biosciences, Falmouth, UK). Embryos were cultured for 7 days at $38.5^{\circ} \mathrm{C}$ in a humidified atmosphere of $5 \% \mathrm{CO}_{2}, 5 \% \mathrm{O}_{2}$, and $90 \% \mathrm{~N}_{2}$. 


\section{Guide-RNA and donor plasmid construction}

The guide-RNA (gRNA) targeting the H11 safe harbor locus on bovine chromosome 17 was designed as previously described [15] (TAGCCATAAGACTACCTAT) and commercially synthesized (Synthego, Redwood City, CA, USA). The donor plasmid construct was designed as previously described [9], containing the endogenous bovine sex-determining region $\mathrm{Y},(S R Y)$ promoter and coding sequence [14], the green fluorescent protein (GFP) coding sequence and SV40 promoter, and $1 \mathrm{~kb}$ homology arms flanked on either side by the CRISPR target site (Fig. 1a). Each piece was commercially synthesized (GeneWiz, LLC, South Plainfield, NJ, USA) and inserted into a pUC19 plasmid using Gibson Assembly Master Mix (New England Biolabs, Inc., Ipswich, MA). Plasmids were clonally amplified using 5-alpha Chemically Competent E. coli (High Efficiency) (New England Biolabs, Inc., Ipswich, MA) and extracted using the EndoFree Plasmid Maxi Kit (Qiagen, Inc., Valencia, CA).

\section{Embryo injection and evaluation}

Approximately 200 in vitro fertilized bovine zygotes were injected approximately $6 \mathrm{~h}$ post insemination using laser assisted cytoplasmic injection [36] with $6 \mathrm{pL}$ of solution containing $67 \mathrm{ng} / \mu \mathrm{L}$ of synthetic gRNA, $167 \mathrm{ng} / \mu \mathrm{L}$ of Cas9 protein (PNA Bio, Inc., Newbury Park, CA) and 133 $\mathrm{ng} / \mu \mathrm{L}$ of donor plasmid. Embryos were then cultured in BO-IVC culture media (IVF Biosciences, Falmouth, UK) for 7 days at $38.5^{\circ} \mathrm{C}$ in a humidified atmosphere of $5 \%$ $\mathrm{CO}_{2}, 5 \% \mathrm{O}_{2}$, and $90 \% \mathrm{~N}_{2}$. On day seven, embryos were scored for developmental stage reach and 22 high grade seven blastocysts were selected and analyzed using fluorescent microscopy on a Nikon Eclipse TE2000-U advanced inverted epifluorescence microscope at $20 \mathrm{X}$ magnification using a filter specific for eGFP fluorescence. Fluorescent images of GFP expressing blastocysts were taken using an Echo Revolve 4 upright, inverted, brightfield microscope at 10X magnification using transillumination for bright field and FITC for GFP expression.

\section{Embryo transfer}

Estrus synchronization was initiated in 15 nulliparous heifers from the Department of Animal Science, University of California, Davis commercial cow herd by inserting an intravaginal progesterone device (1.38 g; Eazi-Breed CIDR; Zoetis) and intramuscular administration of gonadorelin (100 mcg; Factrel; Zoetis) on day 0 (16 days prior to transfer). This number of recipients was chosen with the objective of obtaining at least two SRY knock-in bull calves assuming $60 \%$ response to synchronization, and an expectation that $27 \%$ of recipients receiving conventional in vitro produced (IVP) would result in a live calf. On day 7 , the CIDR was removed and intramuscular prostaglandin (25 mg; Lutalyse; Zoetis) was administered. Recipients were monitored for estrus, and a second intramuscular dose of gonadorelin (100 mcg; Factrel; Zoetis) was administered on day 9. Prior to transfer on day 16, recipient response to synchronization was confirmed via detection of an appropriate corpus luteum with transrectal ultrasonography and nine recipients were deemed suitable for embryo transfer. Prior to transfer, each recipient received a caudal epidural using $100 \mathrm{mg}$ 2\% lidocaine (Xylocaine; Fresenius). A total of nine embryos were transferred via non-surgical, transcervical technique, with each GFP positive blastocyst being deposited into the uterine horn ipsilateral to the corpus luteum. Pregnancy was diagnosed on day 35 of embryonic development by transrectal ultrasonography $(5.0 \mathrm{MHz}$ linear probe; EVO Ibex, E.I. Medical Imaging), and sex was likewise determined at day 68 of development. A single knock-in bull calf was born in April, 2020, and was monitored and maintained at the University of California, Davis Beef Cattle Barn under normal husbandry conditions. The bull calf remains at this facility while he develops to sexual maturity.

\section{DNA extraction and PCR analysis}

Whole blood ( $5 \mathrm{ml}$ ) was collected in EDTA vacutainers (Becton Dickinson) by a veterinarian from the UC Davis veterinary hospital large animal clinic. DNA was extracted either from the buffy coat using the DNeasy Blood and Tissue kit (Qiagen, Inc., Valencia, CA, USA) or from whole blood using red blood cell lysis, SDS/Proteinase K cell lysis, phenol/chloroform/isoamyl alcohol clean up and ethanol precipitation. The placental cotyledon was collected and cut into small pieces. DNA was then extracted from $25 \mathrm{mg}$ of placental cotyledon using the DNeasy Blood and Tissue kit. An ear punch biopsy was taken from the bull calf and used to establish a fibroblast line in culture. Cells were passaged twice in DMEM media (Thermo Fisher, Waltham, MA, USA) containing 10\% fetal bovine serum (Thermo Fisher, Waltham, MA, USA), 1\% Glutamax (Thermo Fisher, Waltham, MA, USA) and 1\% penicillin/streptomycin (Thermo Fisher, Waltham, MA, USA). After the second passage, cells were collected and DNA was extracted using the DNeasy Blood and Tissue kit (Qiagen Inc., Valencia, CA, USA). The target regions were amplified using the polymerase chain reaction (PCR) using primers (F - CCCCAGTGTTGTGCATGTAG; R GTGAATGCCACTGCTGTGTT) for the H11 locus [15] and primers (F - AGGAAGCCAGGAAAGTAA; R CATCCACGTTCTAAGTCTC) for genotypic sexing. The knock-in PCR was performed on a SimpliAmp Thermal Cycler (Applied Biosystems, Foster City, California, USA) with $12.5 \mu \mathrm{L}$ LongAmp Taq 2X Master Mix (New England Biolabs, Inc., Ipswich, MA), $9.5 \mu \mathrm{L}$ of $\mathrm{H}_{2} \mathrm{O}, 1 \mu \mathrm{L}$ of each primer at $10 \mathrm{mM}$ and $1 \mu \mathrm{L}$ of DNA for $5 \mathrm{~min}$ at $94{ }^{\circ} \mathrm{C}, 35$ cycles of $30 \mathrm{~s}$ at $94^{\circ} \mathrm{C}, 30 \mathrm{~s}$ at $60^{\circ} \mathrm{C}$ and $4 \mathrm{~min}$ at $65^{\circ} \mathrm{C}$, followed by $15 \mathrm{~min}$ at $65^{\circ} \mathrm{C}$. The sexing PCR was 
performed on a SimpliAmp Thermal Cycler (Applied Biosystems, Foster City, California, USA) with $12.5 \mu \mathrm{L}$ GoTaq Green Master Mix (Promega Biosciences, LLC, San Luis Obispo, CA, USA), $9.5 \mu \mathrm{L}$ of $\mathrm{H}_{2} \mathrm{O}, 1 \mu \mathrm{L}$ of each primer at $10 \mathrm{mM}$ and $1 \mu \mathrm{L}$ of DNA for $5 \mathrm{~min}$ at $94^{\circ} \mathrm{C}, 35$ cycles of $30 \mathrm{~s}$ at $94^{\circ} \mathrm{C}, 30 \mathrm{~s}$ at $55^{\circ} \mathrm{C}$ and $30 \mathrm{~s}$ at $72^{\circ} \mathrm{C}$, followed by 5 min at $72{ }^{\circ} \mathrm{C}$. Products were visualized on a $1 \%$ agarose gel using a ChemiDoc-ItTS2 Imager (UVP, LLC, Upland, CA), purified using the QIAquick Gel Extraction Kit (Qiagen, Inc., Valencia, CA) and Sanger sequenced (GeneWiz, South Plainfield, NJ).

\section{Whole genome sequencing and mapping}

Genomic DNA extracted from the buffy coat was submitted to Novogene for library construction and whole genome sequencing. Samples were sequenced on an Illumina NovaSeq 6000 sequencer with paired end, $150 \mathrm{bp}$ reads. Raw reads were aligned to the donor plasmid backbone, as well as the predicted knock-in map using Bowtie2-default v2.3.4.1. SAM files were converted to BAM files, sorted and indexed using SAMtools v1.12.0 [37]. Depth was called at each base along the alignment using SAMtools depth v1.12.0 [37].

\section{Assessment of long reads}

Genomic DNA extracted from whole blood was submitted for PacBio long read sequencing in a Sequel II SMRTcell (GeneWiz, USA). Input bam files were converted into FASTA files then assembly-stats (https:// github.com/sanger-pathogens/assembly-stats.git) was used to assess the size of the reads. There were 19,709, 419 reads with total sum length 292,074,095,630 bp which is $\sim 97 \mathrm{x}$ coverage of the bovine genome. The average read length was $14,819.01 \mathrm{bp}$ while the largest read length was $249,262 \mathrm{bp}$.

\section{Identification of candidate long reads}

To identify the reads that had any similarity to the possible inserts, cloning plasmid and/or insertion locus on chromosome 17, a bait file was generated containing:

a) The wild-type locus on Chr17 (1 kb before and after the break point).

b) The pUC19 plasmid backbone.

c) The originally proposed knock in sequence including the homology arms (complete template).

d) The $26 \mathrm{bp}$ allele detected by Sanger sequencing.

Alignment of all input long reads against the bait file using BLASR (5.3.3-SL-release-8.0.0) recruited 314 reads.
Identification of possible structures of edited alleles

To find out the structure of any allele connecting the introduced sequences to the bovine chromosomes, a new reference was generated to include the bovine reference ARS-UCD1.2 [20], together with the pUC19 plasmid and complete template sequences. All candidate reads were aligned against the new reference. To enable better delineation of the allele structure, each read was fragmented into $1 \mathrm{~kb}$ subreads with $0.5 \mathrm{~kb}$ overlap. Read alignments were tested manually and classified into groups that support each allele structure. All suggested structures supported with at least two reads from more than one cluster were considered for further analyses.

\section{Identification of possible junctional sequences between the allele blocks}

The last 50 nucleotides of each edge from the plasmid and proposed complete template were converted into overlapping k-mers of $25 \mathrm{bp}$ length. This way each $50 \mathrm{bp}$ edge was transformed into $26 \mathrm{k}$-mers. Any short read containing at least one of these k-mers or their reverse complement were selected. The k-mer selected reads (660 reads covering the plasmid edges and 3246 reads covering the complete template edges) were error trimmed using Khmer 2 software package. Trimmed reads were assembled into contigs using SSAKE. Contigs were annotated by BLAST alignment against plasmid and complete template sequences and manual examination to identify the novel junction sequences.

\section{Confirmation of the allele sequences}

To further confirm the exact sequence of the three putative alleles, long reads covering each sub-structures of the proposed alleles were subjected to multiple sequence alignment using MAFFT (http://europepmc.org/article/ MED/30976793). To improve the quality of alignment, most of the wild-type sequences were trimmed from the reads. The aligned sequences were used to generate a consensus sequence using the cons tool of EMBOSS package. The consensus sequence was re-aligned using BLAST to the $\mathrm{nr}$ database or the proposed sequences. The full sequence of the three insertion alleles and surrounding bovine genomic sequence are included in Supplementary Materials.

\section{Fluorescence in situ hybridization}

The fibroblast line derived from an ear punch biopsy taken from the bull calf was plated in a T75 flask in DMEM media (Thermo Fisher, Waltham, MA, USA) containing 10\% fetal bovine serum (Thermo Fisher, Waltham, MA, USA), 1\% Glutamax (Thermo Fisher, Waltham, MA, USA) and $1 \%$ penicillin/streptomycin (Thermo Fisher, Waltham, MA, USA). Once the cells reached $\sim 80 \%$ confluency, $1.25 \%$ Gibco KaryoMAX Colcemid Solution 
(Thermo Fisher, Waltham, MA, USA) was added to the media and incubated at $37^{\circ} \mathrm{C}$ and $5 \% \mathrm{CO}_{2}$ for $1 \mathrm{~h}$. Cell were then collected, resuspended in $10 \mathrm{~mL}$ of $0.56 \% \mathrm{KCl}$ hypotonic solution and incubated at $37^{\circ} \mathrm{C}$ for $10 \mathrm{~min}$. Cells were then fixed in a 3:1 methanol to glacial acetic acid solution at $4{ }^{\circ} \mathrm{C}$ for 1 week. Once fixed, three to four drops of cell solution were applied to slides at a $45^{\circ}$ angle and allowed to air dry. Slides were then hardened, i.e., aged at $-20^{\circ} \mathrm{C}$ for 1 week. Slides were then used for fluorescence in situ hybridization as previously described [38, 39] using Roche DIG-Nick Translation Kit and AntiDigoxigenin-Fluorescein, Fab fragments (Roche Applied Science, Upper Bavaria, Germany) to label the plasmid containing SRY. The BTA 17 chromosome-specific BAC (CHORI 371i17) with a centromere proximal location (15, 482,193 - 15,677,551) was labelled using Red dUTP (Abbott Molecular, Des Plaines, IL) and a direct Nick Translation Kit (Abbott Molecular). Chromosomes were visualized using Vectashield mounting media with DAPI (Abcam, Cambridge, MA, USA). Mitotic metaphase chromosomes were examined, and images collected using an Olympus BX41 epifluorescence microscope equipped with an automatic filter wheel (Chroma Technology 82,000, DAPI/FITC/TRITC filter set), X-cite 120 Series metalhalide fiber optic lamp and Applied Imaging software (CytoVision version 7.4 GENUS, Leica Biosystems).

\section{Supplementary Information}

The online version contains supplementary material available at https://doi. org/10.1186/s12864-021-07418-3

Additional file 1.

Additional file 2 .

\section{Acknowledgments}

The authors would sincerely like to thank Dr. Tara Urbano, who was instrumental in monitoring the health of the animals and Donald Harper, Marissa Fisher, Danny Vickers and the UC Davis Beef Barn residents for excellent management of the animal herds. We would also like to thank Ashley Young and Rebecca Ozeran for collection of ovaries, and Erika Paulson for assistance with embryo injections.

\section{Authors' contributions}

JRO and SLH performed the experiments with additional input from JDM, PJR and ALV. JRO, SLH, JCL,AEY and JFT participated in sample processing and analysis. JRO and TAM performed the bioinformatic analysis. JRO and MED designed the FISH experiments, JRO and JMS performed the FISH experiments and JRO and MED analyzed and interpreted the results. BRM was responsible for embryo transfers and monitoring of pregnancies. JRO, TAM, JDM, PJR and ALV wrote the manuscript with suggestions from all COauthors. The author(s) read and approved the final manuscript.

\section{Funding}

This project was supported by Biotechnology Risk Assessment Grant Program competitive grant no. 2015-33522-24106 from the U.S. Department of Agriculture, the Academic Federation Innovation Development Award at UC Davis, the Russell L. Rustici Rangeland and Cattle Research Endowment in the College of Agricultural and Environmental Science at UC Davis, the California Agricultural Experiment Station of the University of California, Davis, the Henry A. Jastro Research Fellowship in the College of Agricultural and Environmental Science at UC Davis and the National Institute for Food and Agriculture National Needs Graduate and Postgraduate Fellowship no. 2017-38420-26790 from the U.S. Department of Agriculture.

\section{Availability of data and materials}

Raw sequence reads from Illumina NovaSeq and PacBio Sequel II sequencing are available in the NCBI Sequence Read Archive as BioProject PRJNA625124, BioSample SAMN14593732 and SRA accession number SRR12005315 SRR12005330.

\section{Ethics approval and consent to participate}

All the experiments carried out with the use of animals were approved and performed in accordance with the University of California, Davis Institutional Animal Care and Use Committee (IACUC) approved protocol \#20595. This institution is accredited by the Association for Assessment and Accreditation of Laboratory Animal Care, International (AAALAC). This institution has an Animal Welfare Assurance on file with the Office of Laboratory Animal Welfare (OLAW). The Assurance Number is D16-00272 (A3433-01). The IACUC is constituted in accordance with U.S. Public Health Service (PHS) Animal Welfare Policy and includes a member of the public and a nonscientist.

\section{Consent for publication}

Not applicable.

\section{Competing interests}

The authors declare that they have no competing interests.

\section{Author details}

${ }^{1}$ Department of Animal Science, University of California - Davis, Davis, CA, USA. ${ }^{2}$ Department of Population Health and Reproduction, School of Veterinary Medicine, University of California - Davis, Davis, CA, USA.

${ }^{3}$ Department of Clinical Pathology, School of Medicine, University of Mansoura, Mansoura, Egypt.

Received: 29 December 2020 Accepted: 31 January 2021 Published online: 12 February 2021

\section{References}

1. Hammer RE, Pursel VG, Rexroad CE Jr, Wall RJ, Bolt DJ, Ebert KM, Palmiter RD, Brinster RL. Production of transgenic rabbits, sheep and pigs by microinjection. Nature. 1985;315:680-3.

2. Tan W, Proudfoot C, Lillico SG, Whitelaw CB. Gene targeting, genome editing: from Dolly to editors. Transgenic Res. 2016;25:273-87.

3. Mehravar M, Shirazi A, Nazari M, Banan M. Mosaicism in CRISPR/Cas9mediated genome editing. Dev Biol. 2019:445:156-62.

4. Danner E, Bashir S, Yumlu S, Wurst W, Wefers B, Kuhn R. Control of gene editing by manipulation of DNA repair mechanisms. Mamm Genome. 2017: 28:262-74.

5. Hustedt N, Durocher D. The control of DNA repair by the cell cycle. Nat Cell Biol. 2016;19:1-9.

6. Mclean Z, Oback B, Laible G. Embryo-mediated genome editing for accelerated genetic improvement of livestock. Front Agricultural Science and Engineering. 2020

7. Yao X, Wang X, Hu X, Liu Z, Liu J, Zhou H, Shen X, Wei Y, Huang Z, Ying W, et al. Homology-mediated end joining-based targeted integration using CRISPR/Cas9. Cell Res. 2017:27:801-14

8. Xie L, Sun J, Mo L, Xu T, Shahzad Q, Chen D, Yang W, Liao Y, Lu Y. HMEJmediated efficient site-specific gene integration in chicken cells. J Biol Eng. 2019;13:90.

9. Owen JR, Hennig SL, Paulson EE, Lin JL, Ross PJ, Van Eenennaam AL. Improved rate of targeted gene knock-in of in-vitro fertilized bovine embryos. Proceedings of the Association for the Advancement of Animal Breeding and Genetics. 2019;23:7-10.

10. Owen JR, Hennig SL, McNabb BR, Lin JC, Young AE, Murray JD, Ross PJ, Van Eenennaam AL. Harnessing endogenous repair mechanisms for targeted gene knock-in of bovine embryos. Sci Rep. 2020;10:16031.

11. Makela JA, Koskenniemi JJ, Virtanen HE, Toppari J. Testis Development. Endocrinology Review. 2019;40:857-905.

12. Vilarino M, Suchy FP, Rashid ST, Lindsay H, Reyes J, McNabb BR, van der Meulen T, Huising MO, Nakauchi H, Ross PJ. Mosaicism diminishes the value 
of pre-implantation embryo biopsies for detecting CRISPR/Cas9 induced mutations in sheep. Transgenic Res. 2018;27:525-37.

13. Lamas-Toranzo I, Galiano-Cogolludo B, Cornudella-Ardiaca F, CobosFigueroa J, Ousinde O, Bermejo-Álvarez P. Strategies to reduce genetic mosaicism following CRISPR-mediated genome edition in bovine embryos. Sci Rep. 2019:9:14900.

14. Daneau I, Houde A, Ethier JF, Lussier JG, Silversides DW. Bovine SRY gene locus: cloning and testicular expression. Biol Reprod. 1995;52:591-9.

15. Hennig SL, Owen JR, Lin JC, Young AE, Ross PJ, Van Eenennaam AL, Murray JD. Evaluation of mosaicism and off-target mutations in CRISPR-mediated genome edited bovine embryos. Sci Rep. 2020;10:22309.

16. Ruan J, Li H, Xu K, Wu T, Wei J, Zhou R, Liu Z, Mu Y, Yang S, Ouyang H, et al. Highly efficient CRISPR/Cas9-mediated transgene knockin at the H11 locus in pigs. Sci Rep. 2015;5:14253.

17. Papapetrou EP, Schambach A. Gene insertion into genomic safe harbors for human gene therapy. Mol Ther. 2016;24:678-84.

18. Young AE, Mansour TA, McNabb BR, Owen JR, Trott JF, Brown CT, Van Eenennaam AL. Genomic and phenotypic analyses of six offspring of a genome-edited hornless bull. Nat Biotechnol. 2020;38:225-32.

19. Norris AL, Lee SS, Greenlees K, Tadesse DA, Miller MF, Lombardi HA. Template plasmid integration in germline genome-edited cattle. Nat Biotechnol. 2020;38:163-4.

20. Rosen BD, Bickhart DM, Schnabel RD, Koren S, Elsik CG, Tseng E, Rowan TN, Low WY, Zimin A, Couldrey C, et al. De novo assembly of the cattle reference genome with single-molecule sequencing. Gigascience. 2020;9.

21. Ealy AD, Wooldridge LK, McCoski SR. BOARD INVITED REVIEW: post-transfer consequences of in vitro-produced embryos in cattle. J Anim Sci. 2019;97: 2555-68.

22. Bordignon V, Keyston R, Lazaris A, Bilodeau AS, Pontes JH, Arnold D, Fecteau G, Keefer C, Smith LC. Transgene expression of green fluorescent protein and germ line transmission in cloned calves derived from in vitrotransfected somatic cells. Biol Reprod. 2003;68:2013-23.

23. Bishop TF, Van Eenennaam AL. Genome editing approaches to augment livestock breeding programs. J Exp Biol. 2020;223.

24. Wei J, Wagner S, Maclean P, Brophy B, Cole S, Smolenski G, Carlson DF, Fahrenkrug SC, Wells DN, Laible G. Cattle with a precise, zygote-mediated deletion safely eliminate the major milk allergen beta-lactoglobulin. Sci Rep. 2018;8:7661

25. Suzuki K, Tsunekawa Y, Hernandez-Benitez R, Wu J, Zhu J, Kim EJ, Hatanaka F, Yamamoto M, Araoka T, Li Z, et al. In vivo genome editing via CRISPR/ Cas9 mediated homology-independent targeted integration. Nature. 2016; 540:144-9.

26. Jiang W, Liu L, Chang Q, Xing F, Ma Z, Fang Z, Zhou J, Fu L, Wang H, Huang $X$, et al. Production of Wilson disease model rabbits with homologydirected precision point mutations in the ATP7B gene using the CRISPR/ Cas9 system. Sci Rep. 2018;8:1332.

27. Kumita W, Sato K, Suzuki Y, Kurotaki Y, Harada T, Zhou Y, Kishi N, Sato K, Aiba A, Sakakibara Y, et al. Efficient generation of Knock-in/Knock-out marmoset embryo via CRISPR/Cas9 gene editing. Sci Rep. 2019;9:12719.

28. Menchaca A, Dos Santos-Neto PC, Souza-Neves M, Cuadro F, Mulet AP, Tesson L, Chenouard V, Guiffes A, Heslan JM, Gantier M, et al. Otoferlin gene editing in sheep via CRISPR-assisted SsODN-mediated homology directed repair. Sci Rep. 2020;10:5995.

29. Quadros RM, Miura H, Harms DW, Akatsuka H, Sato T, Aida T, Redder R, Richardson GP, Inagaki Y, Sakai D, et al. Easi-CRISPR: a robust method for one-step generation of mice carrying conditional and insertion alleles using long ssDNA donors and CRISPR ribonucleoproteins. Genome Biol. 2017;18: 92.

30. Remy S, Chenouard V, Tesson L, Usal C, Menoret S, Brusselle L, Heslan JM, Nguyen TH, Bellien J, Merot J, et al. Generation of gene-edited rats by delivery of CRISPR/Cas9 protein and donor DNA into intact zygotes using electroporation. Sci Rep. 2017;7:16554.

31. Mariati HSCL, JHM Y, Fang SG, Yang Y. Impact of using different promoters and matrix attachment regions on recombinant protein expression level and stability in stably transfected CHO cells. Mol Biotechnol. 2015;57:138-44.

32. Gim G-M, Kwon D-H, Lee W-W, Jung D-J, Kim D-H, Yi J-K, Jang G. Transgenic F2 bovine embryos show stable germline transmission and maintenance of transgene expression through two generations. Bio Reprod. 2020;103:1148-51.

33. FDA (United States Food and Drug Administration): Guidance for Industry \#187, Regulation of Intentionally Altered Genomic DNA in Animals. https://www.fdagov/regulatory-information/search-fda-guidance-documents/ cvm-gfi-187-regulation-intentionally-altered-genomic-dna-animals 2017.

34. Van Eenennaam AL, Wells KD, Murray JD. Proposed U.S. regulation of geneedited food animals is not fit for purpose. NPJ Science of Food. 2019;3:3

35. Bakhtari A, Ross PJ. DPPA3 prevents cytosine hydroxymethylation of the maternal pronucleus and is required for normal development in bovine embryos. Epigenetics. 2014;9:1271-9.

36. Bogliotti YS, Vilarino M, Ross PJ: Laser-assisted Cytoplasmic Microinjection in Livestock Zygotes. Journal of Visualized Experiments 2016.

37. Li H, Handsaker B, Wysoker A, Fennell T, Ruan J, Homer N, Marth G, Abecasis G, Durbin R. Genome project data processing S: the sequence alignment/ map format and SAMtools. Bioinformatics. 2009;25:2078-9.

38. McPherson MC, Robinson CM, Gehlen LP, Delany ME. Comparative cytogenomics of poultry: mapping of single gene and repeat loci in the Japanese quail (Coturnix japonica). Chromosom Res. 2014;22:71-83.

39. O'Hare TH, Delany ME. Genetic variation exists for telomeric array organization within and among the genomes of normal, immortalized, and transformed chicken systems. Chromosom Res. 2009;17:947-64.

\section{Publisher's Note}

Springer Nature remains neutral with regard to jurisdictional claims in published maps and institutional affiliations.

\section{Ready to submit your research? Choose BMC and benefit from:}

- fast, convenient online submission

- thorough peer review by experienced researchers in your field

- rapid publication on acceptance

- support for research data, including large and complex data types

- gold Open Access which fosters wider collaboration and increased citations

- maximum visibility for your research: over $100 \mathrm{M}$ website views per year

At BMC, research is always in progress.

Learn more biomedcentral.com/submissions 